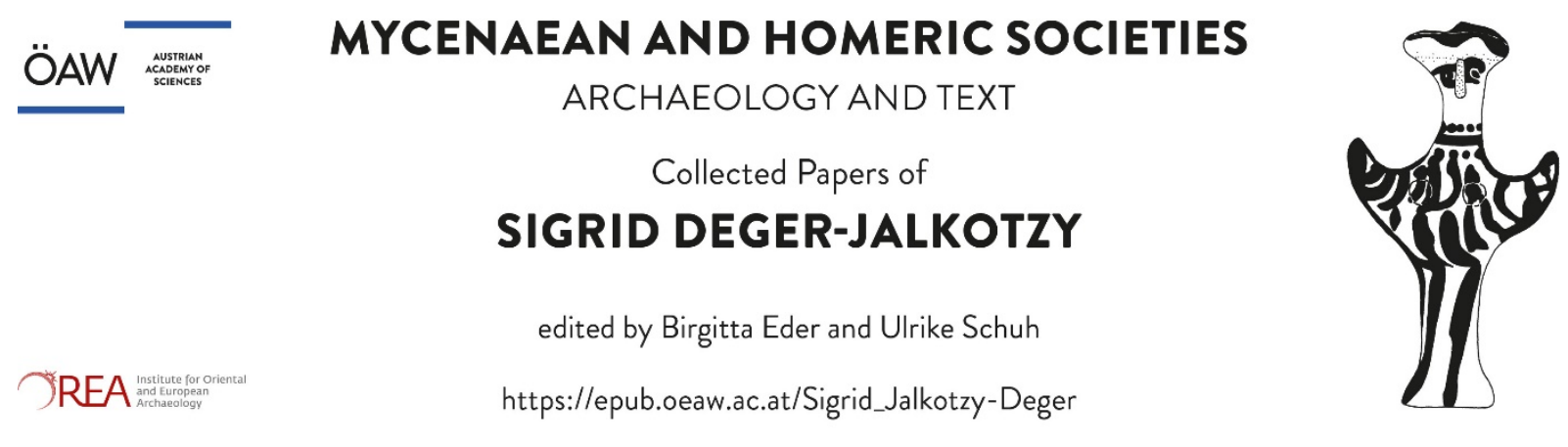

24.

S. Deger-Jalkotzy - Ph. Dakoronia

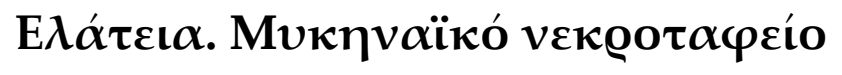

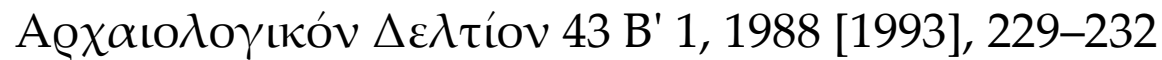

(c)

mit freundlicher Genehmigung / with kind permission

Dieses Dokument darf ausschließlich für wissenschaftliche Zwecke genutzt werden (Lizenz CC BY-NC-ND), gewerbliche Nutzung wird urheberrechtlich verfolgt.

This document is for scientific use only (license CC BY-NC-ND), commercial use of copyrighted material will be prosecuted. 


\section{YПOYРГЕIO ПОАITILMOY}

\section{APXAIO $\Lambda$ OГIKON $\triangle \mathrm{E} \Lambda \mathrm{TION}$}

\section{TOMO乏 43 (1988) \\ MEPO $B^{\prime} 1$ - XPONIKA}

Myrenische Kommission der osterreicivichs: A. -afomio der Wissenscina!t:n

1010 Wien, Or. Ignaz Seipel-F!atz 2

Mon. Kh. 103/20 
EПIऽTHMONIKH EПITPOחH

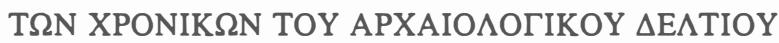

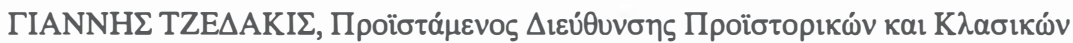

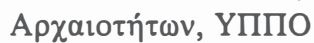

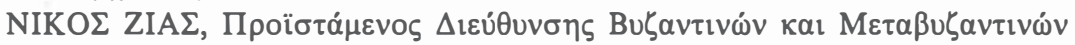

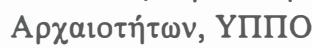

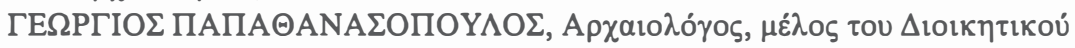

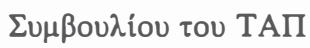

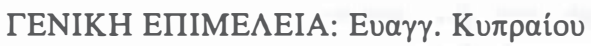

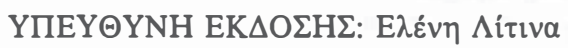

$\Delta \mathrm{IOP} \Omega \Omega \Sigma \mathrm{EI} \Sigma:$ E $\lambda \varepsilon \dot{v} \eta \Lambda$ ítıva

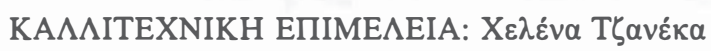

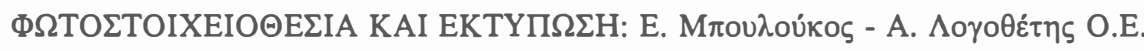

๑ Т TAMEIO APXAIO $\Lambda$ OГIK $\Omega N$ ПOP $\Omega N$ KAI AПA $\Lambda$ OTPI $\Omega \Sigma E \Omega N$ $\triangle$ IEY $\Theta Y N \Sigma H ~ \triangle H M O \Sigma I E Y M A T \Omega N$

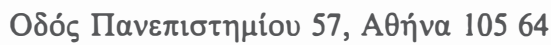

ISSN 0570-622X 


\section{ПEPIEXOMENA}

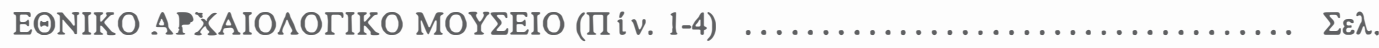

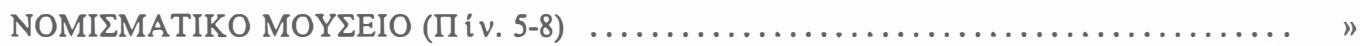

ЕПIГРАФІКО MOYГЕIO

BYZANTINO KAI XPIITIANIKO MOYLEIO

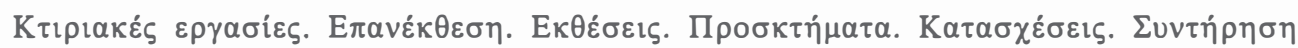

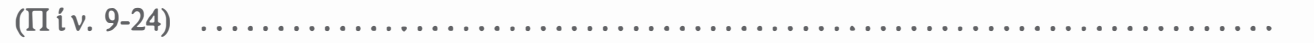

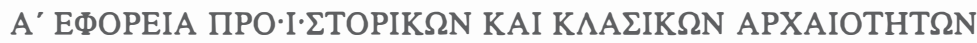

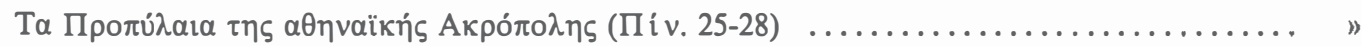

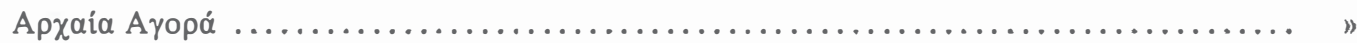

$\Gamma^{\prime}$ ЕФOPEIA ПРО $\cdot \cdot \Sigma T O P I K \Omega N$ KAI K $\Lambda$ A $\Sigma I K \Omega N$ APXAIOTHT $\Omega N$

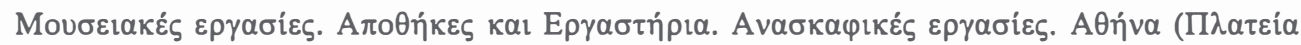

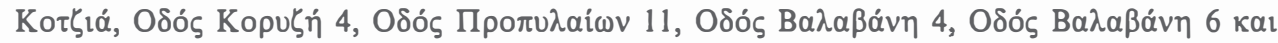

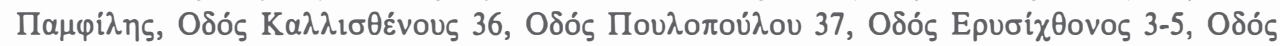

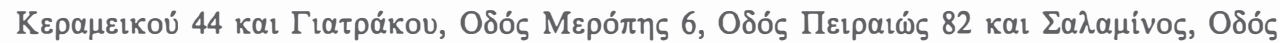

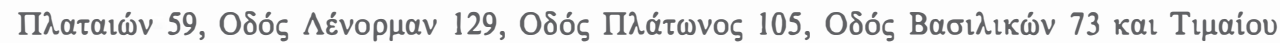

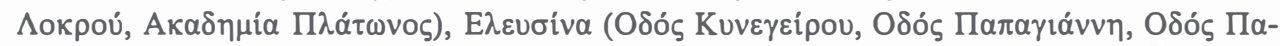

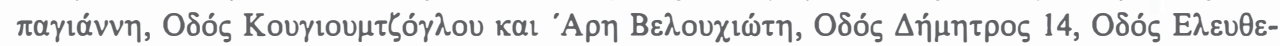

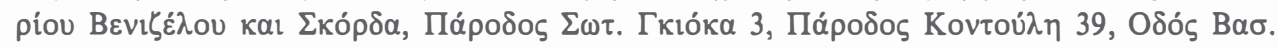

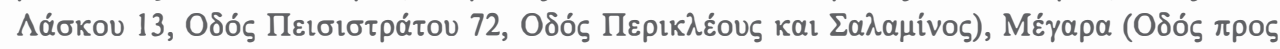

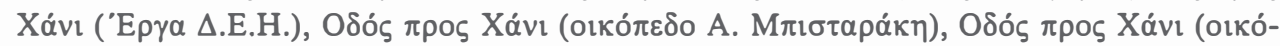

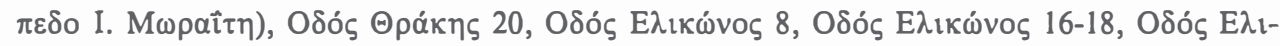

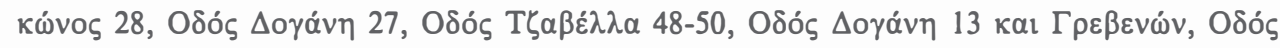

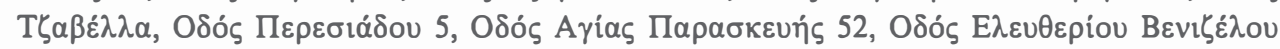

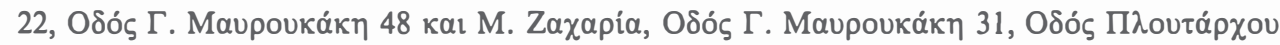

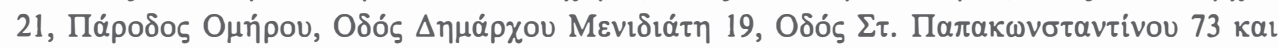

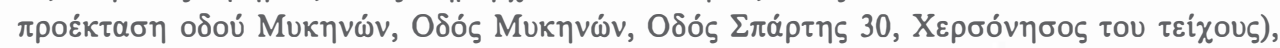

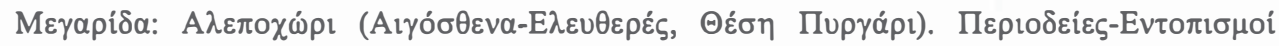

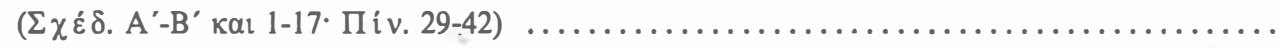

\section{B' ЕФOPEIA ПРО·I· $\Sigma$ TOPIK $\Omega$ N KAI K $\Lambda$ A $\Sigma I K \Omega N$ APXAIOTHT $\Omega N$}

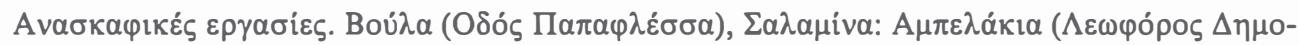

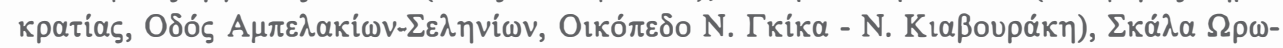

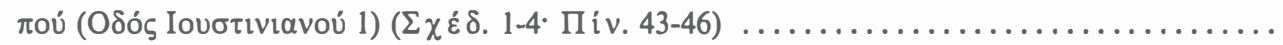

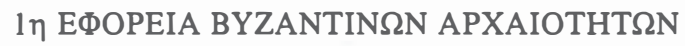

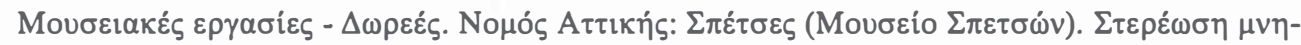

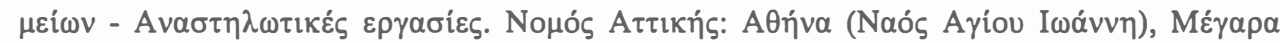

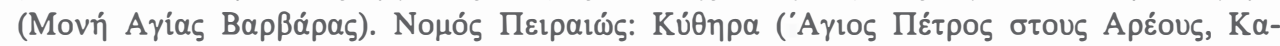

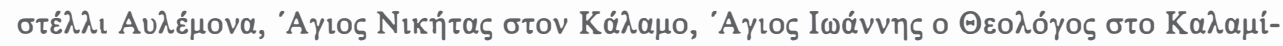

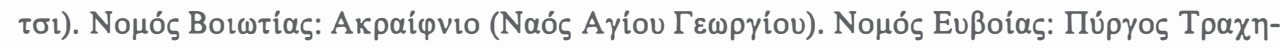

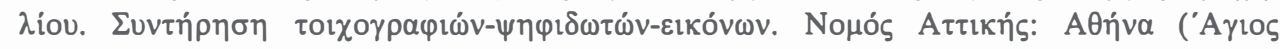

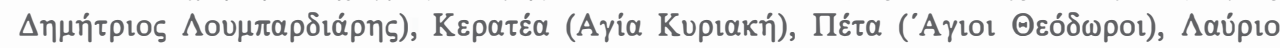

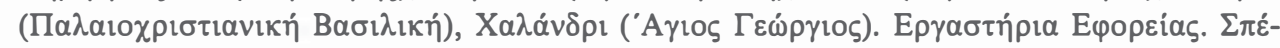

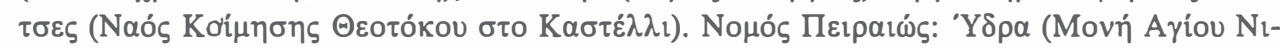

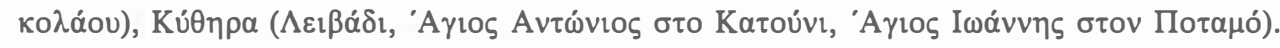

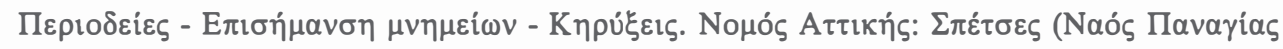




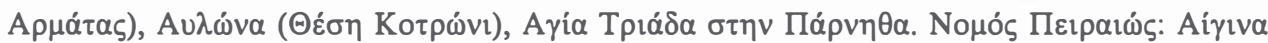
('A

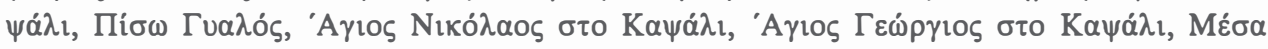

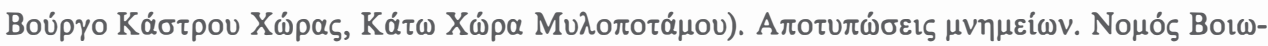

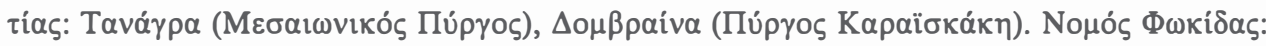

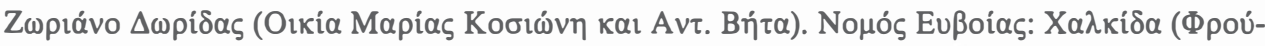

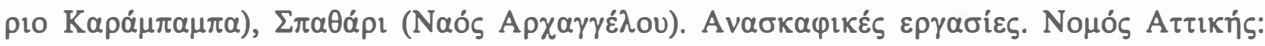

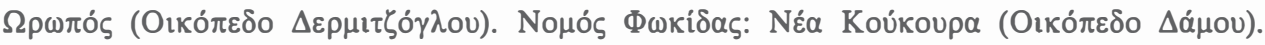

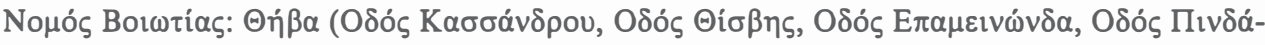

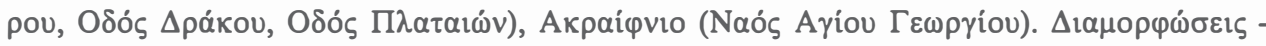

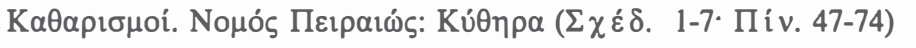

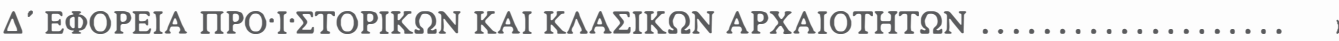

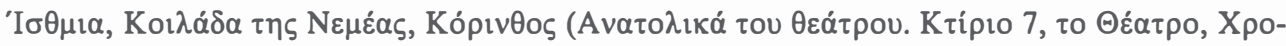

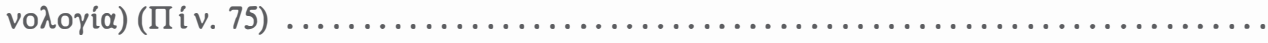

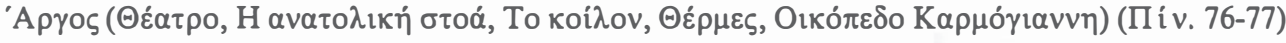

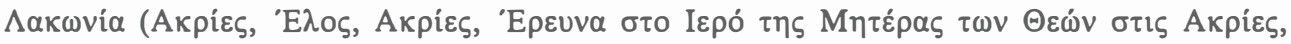

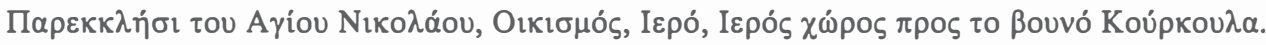

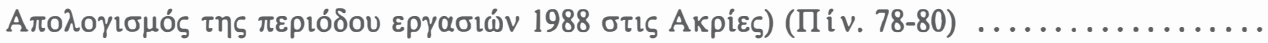

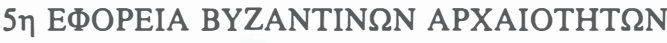

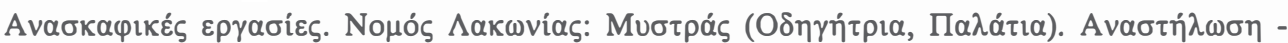

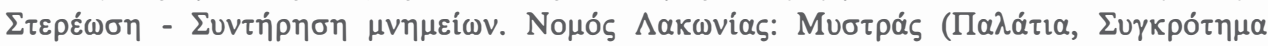

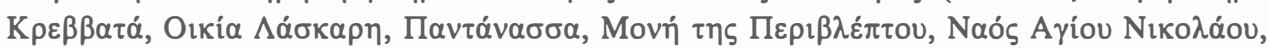

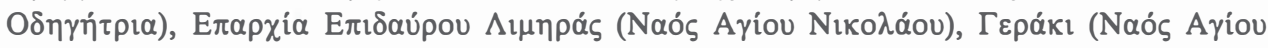

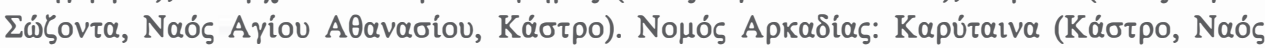

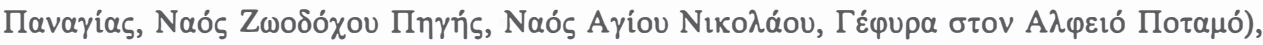

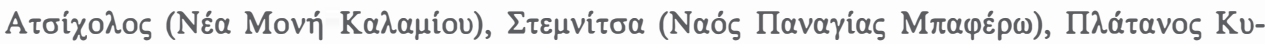

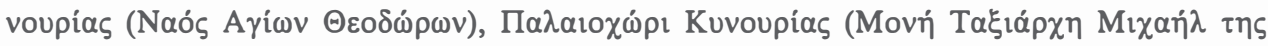

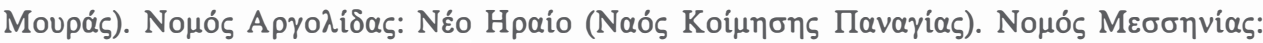

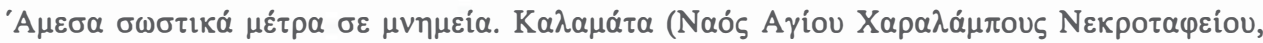

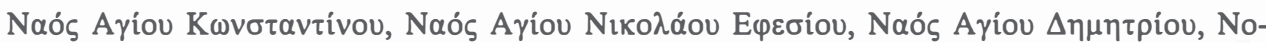

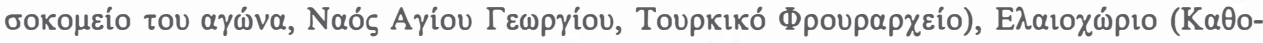

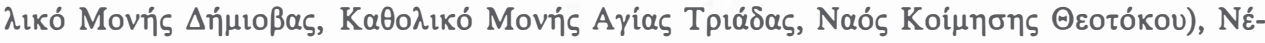

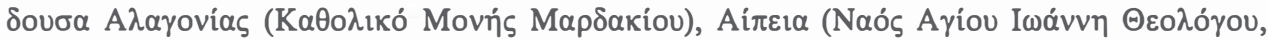

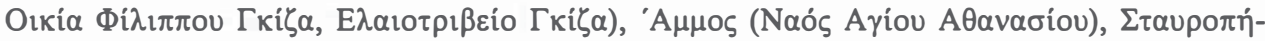

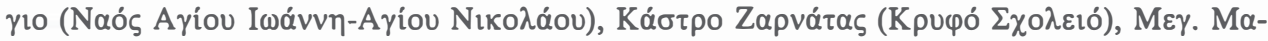

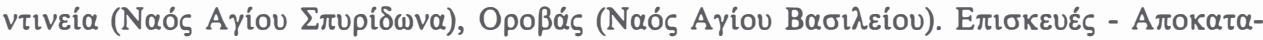

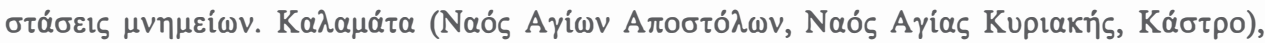

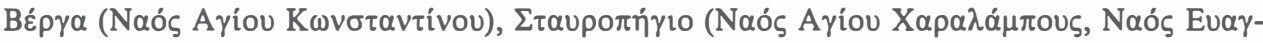

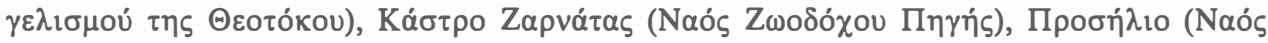

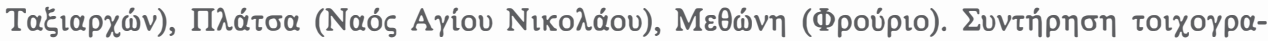

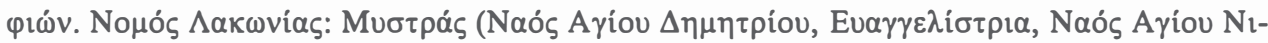

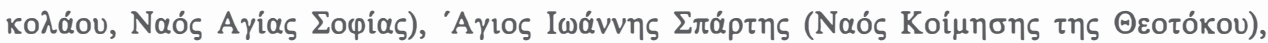

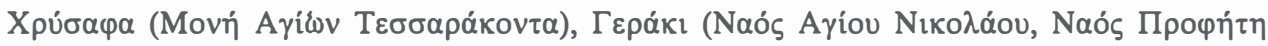

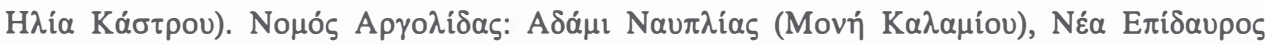

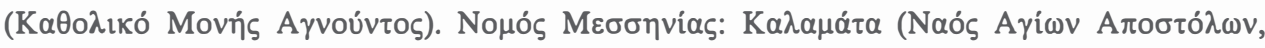

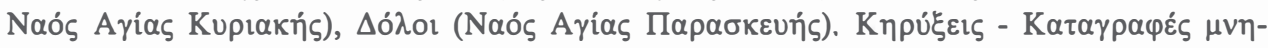

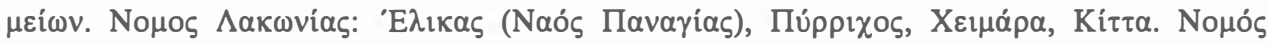

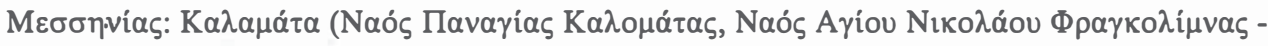




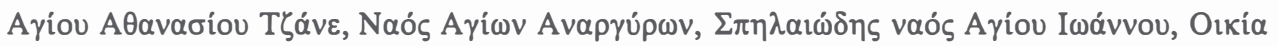

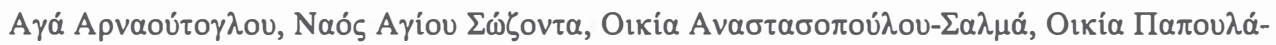

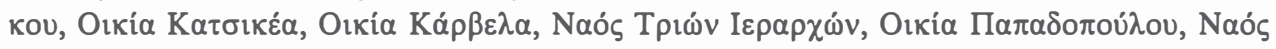

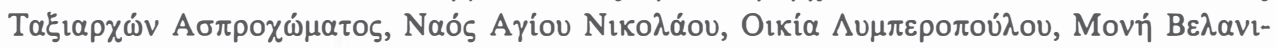

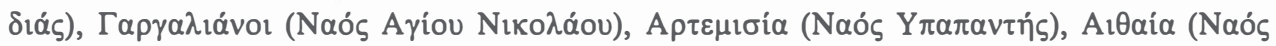

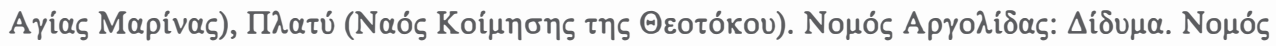

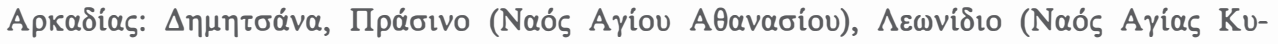

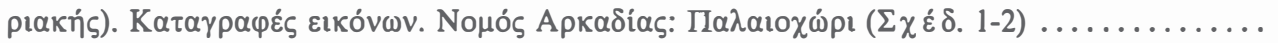

$\Sigma \varepsilon \lambda . \quad 116-141$

\section{Z' ЕФОРЕIA ПРО·I· $\Sigma$ TOPIK $\Omega$ N KAI K $\Lambda$ A $\Sigma I K \Omega N$ APXAIOTHT $\Omega N$}

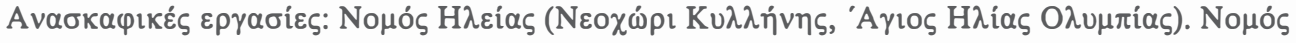

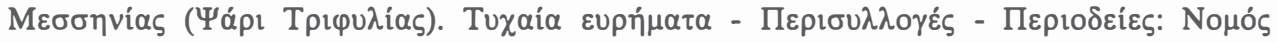

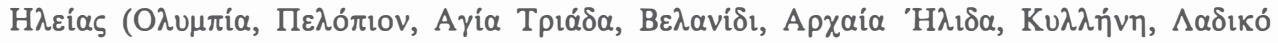

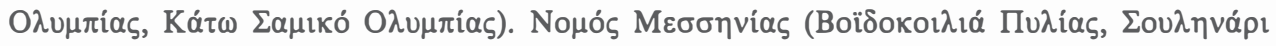

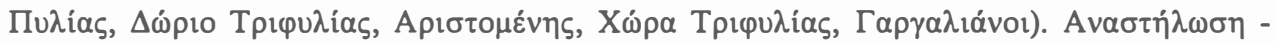

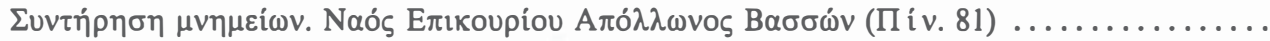

\section{$\Sigma T^{\prime}$ ЕФОРЕIA ПРО·І· $\Sigma$ TOPIK $\Omega$ N KAI K $\Lambda$ A $\Sigma I K \Omega N$ APXAIOTHT $\Omega N$}

Nopó

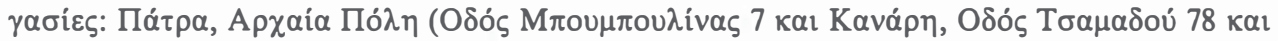

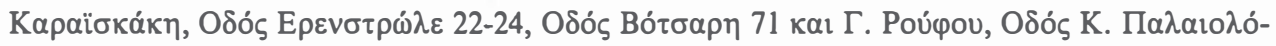

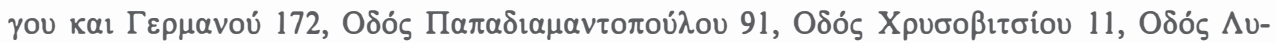

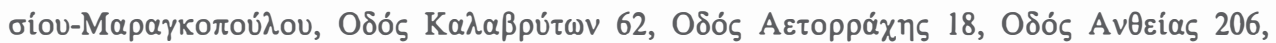

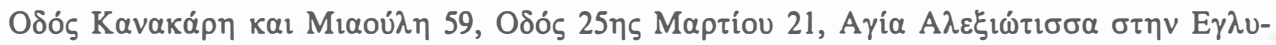

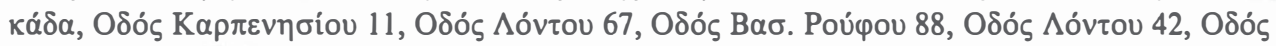

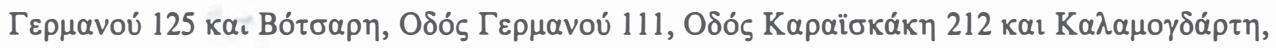

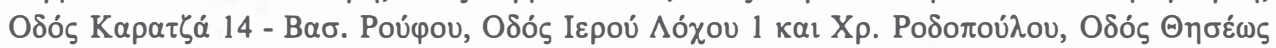

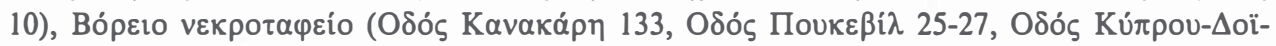

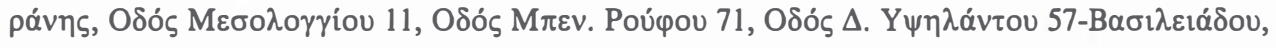

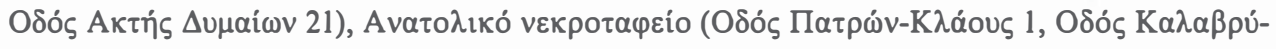

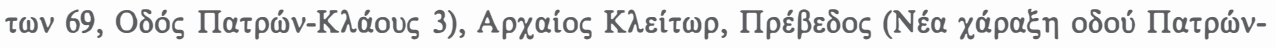

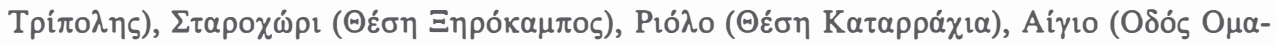

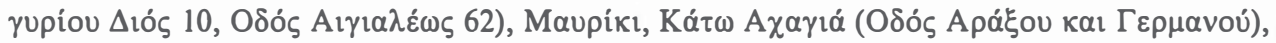

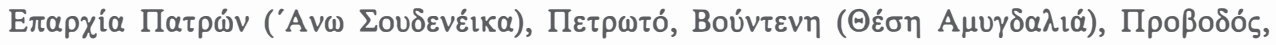

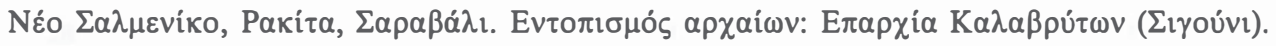

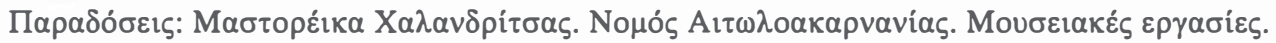

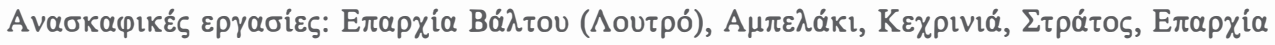

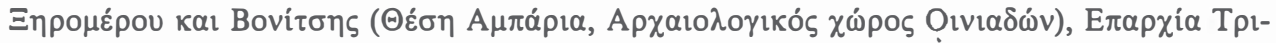

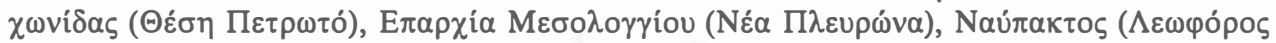

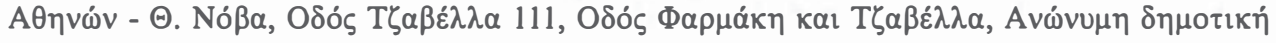

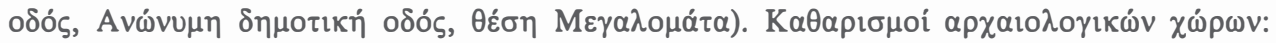

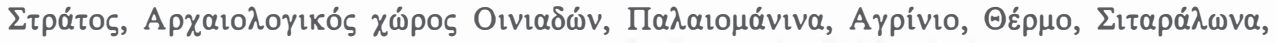

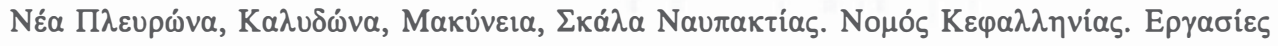

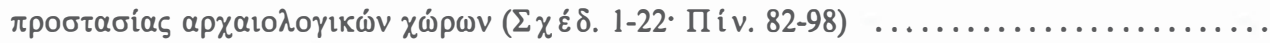

\section{$6 \eta$ EФОРЕIA BYZANTIN $\Omega N$ APXAIOTHT $\Omega N$}

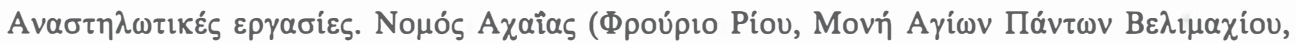

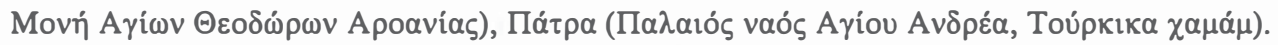

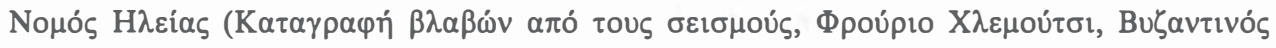

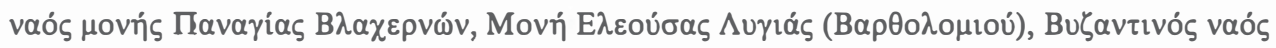

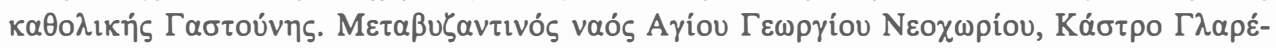

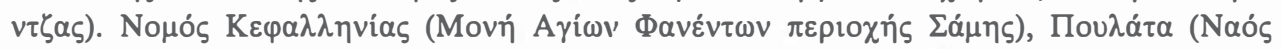




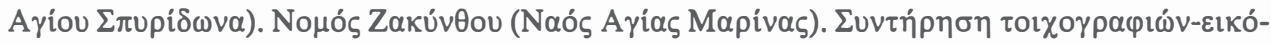

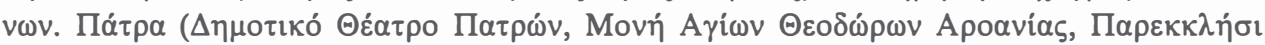

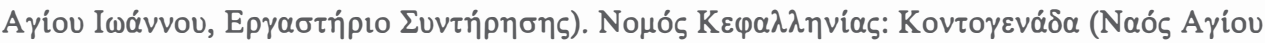

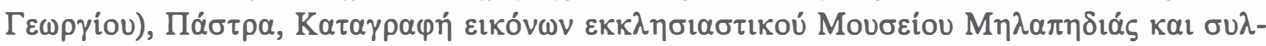

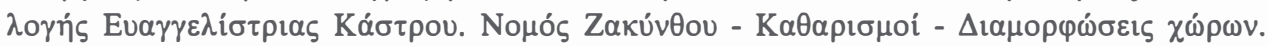

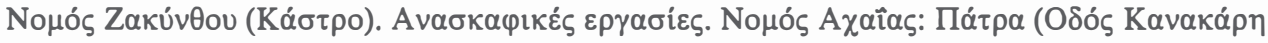

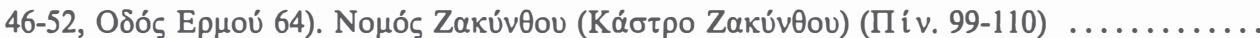

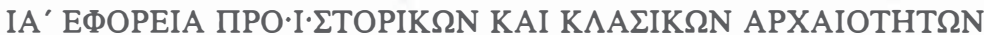

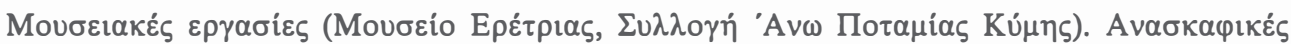

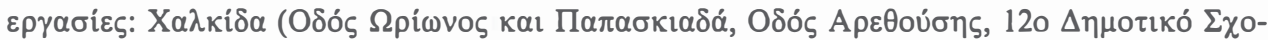

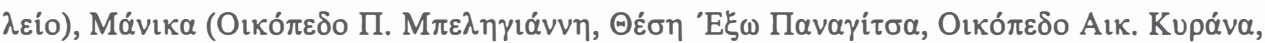

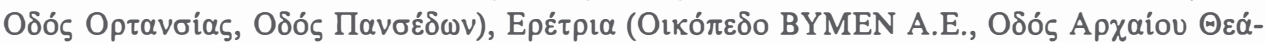

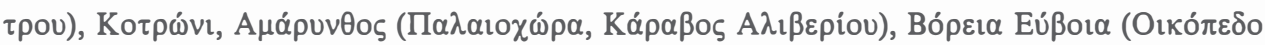

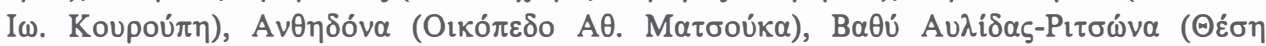

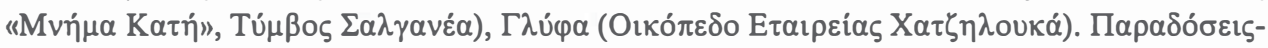

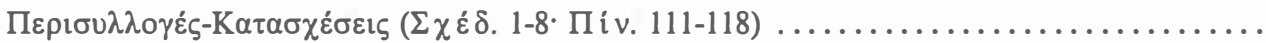

\section{I $\Delta^{\prime}$ ЕФОРЕIA ПРО $\cdot\ulcorner\cdot \Sigma T O P I K \Omega N$ KAI K $\Lambda$ A $\Sigma I K \Omega N$ APXAIOTHT $\Omega N$}

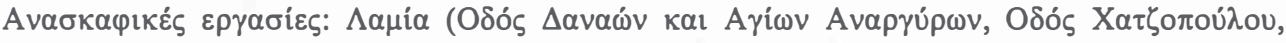

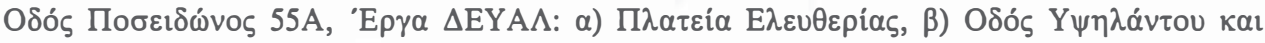

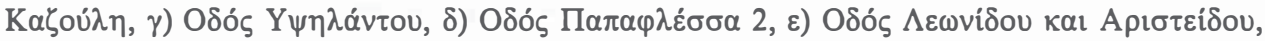

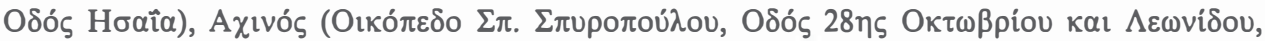

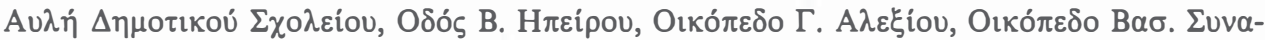

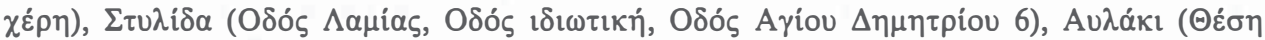

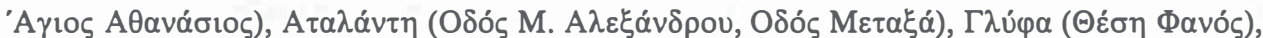

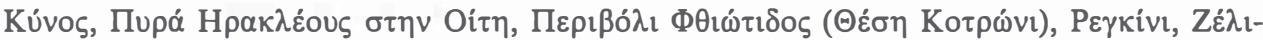

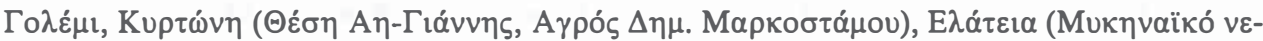

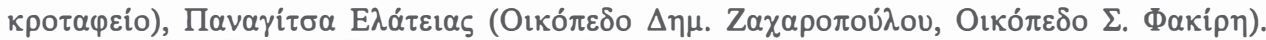

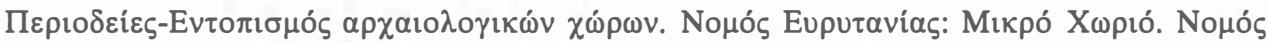

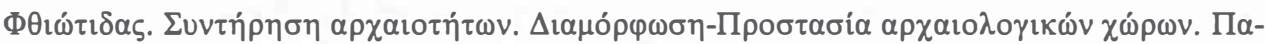

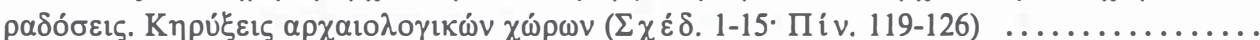

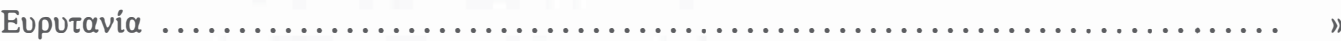

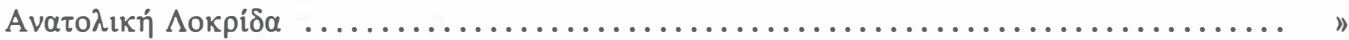

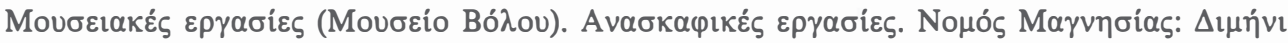

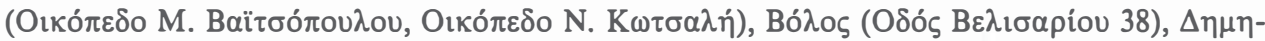

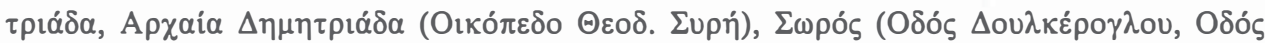

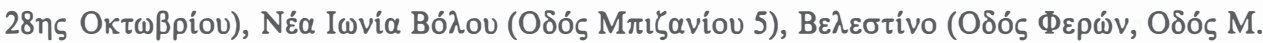

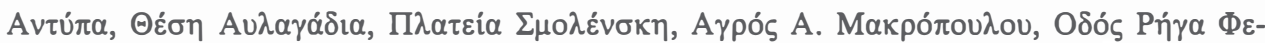

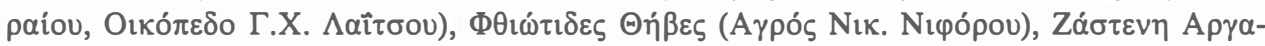

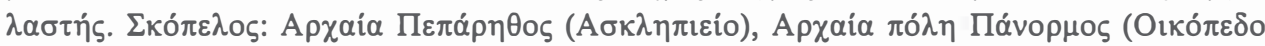

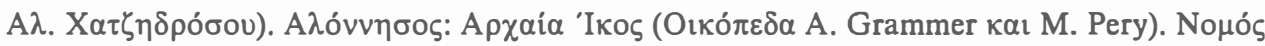

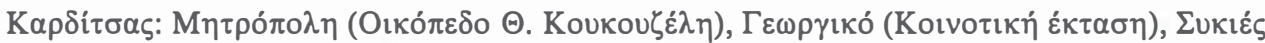




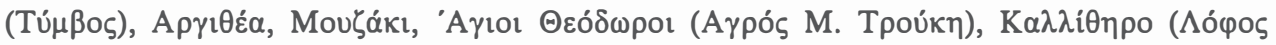

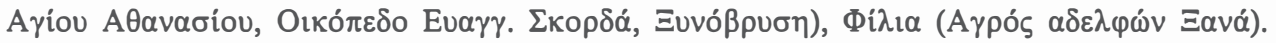

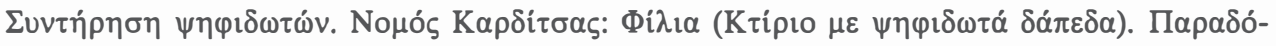

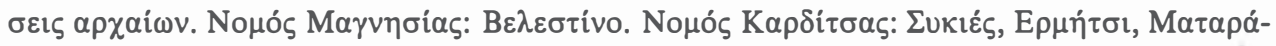

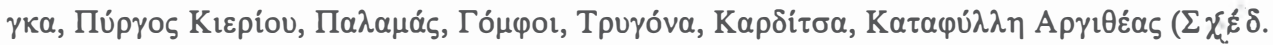

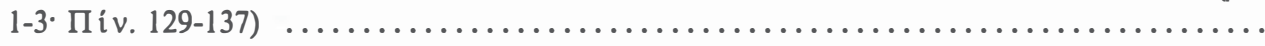

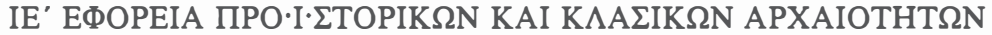

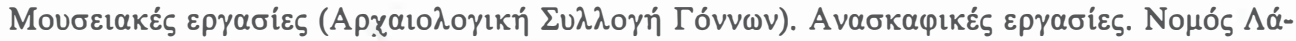

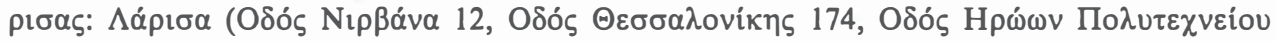

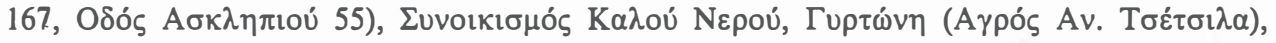

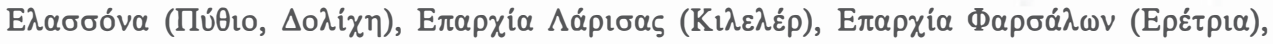

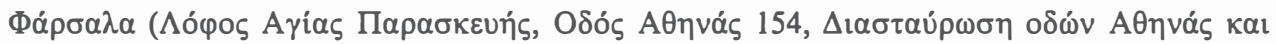

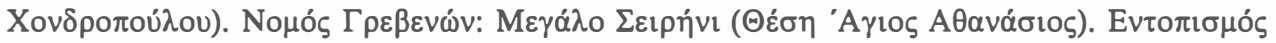

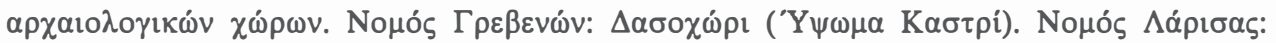

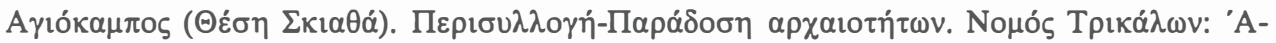

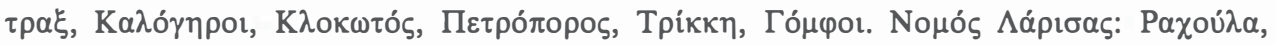

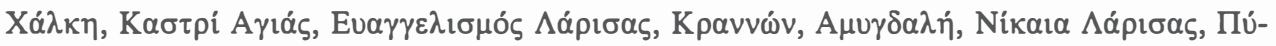

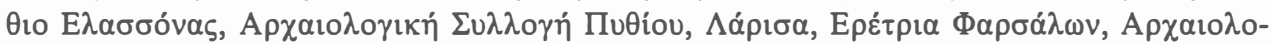

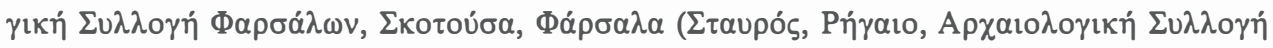

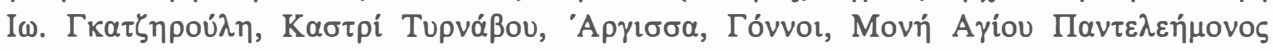

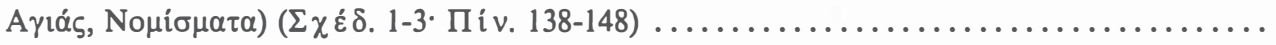

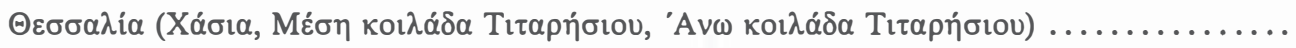

$\Gamma \rho \varepsilon \beta \varepsilon v \alpha ́$. . .

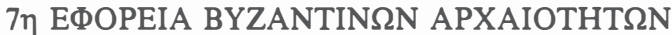

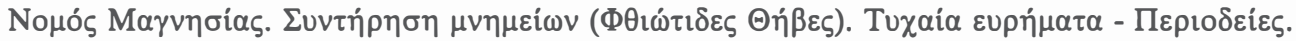

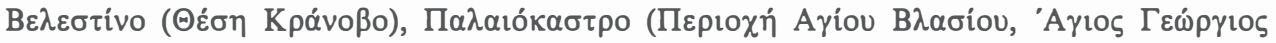

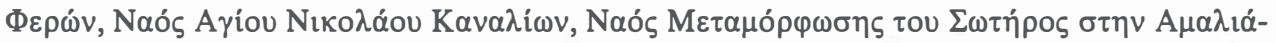

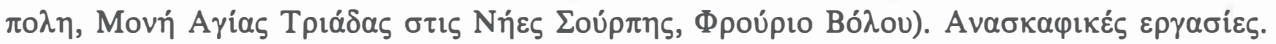

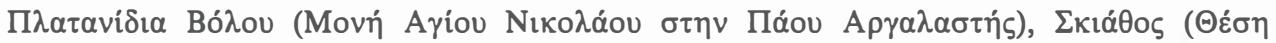

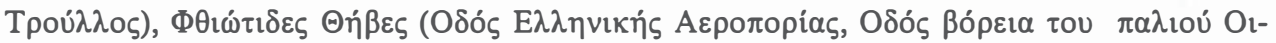

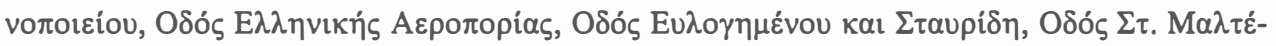

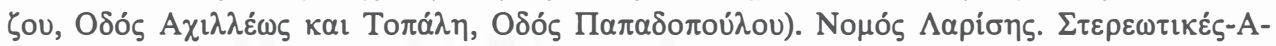

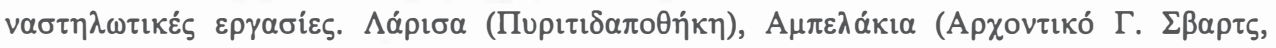

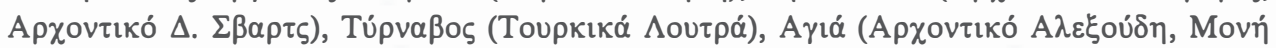

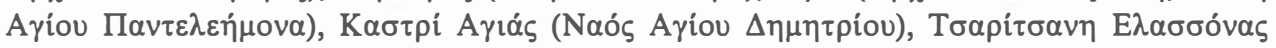

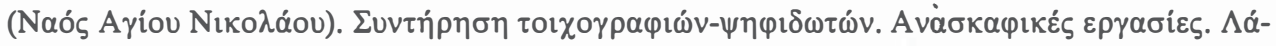

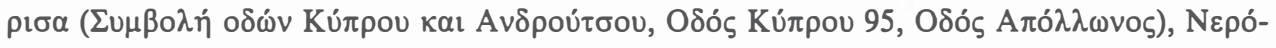

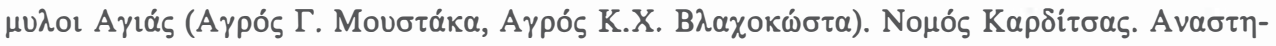

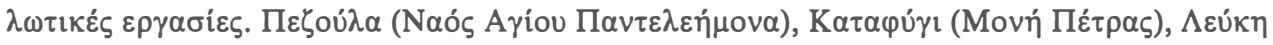

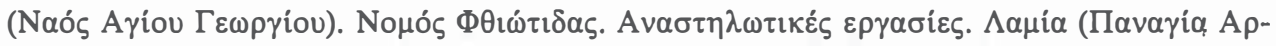

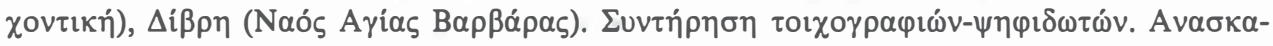

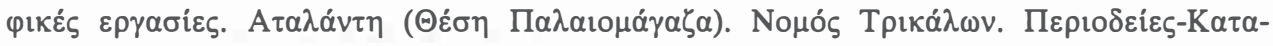

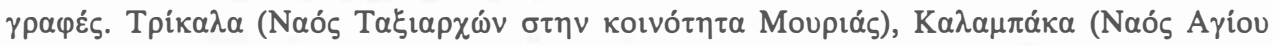

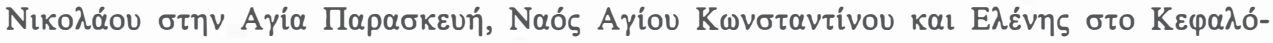

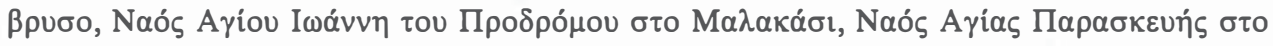

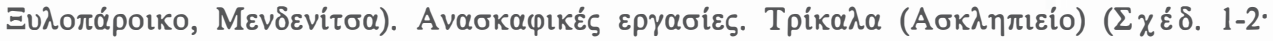

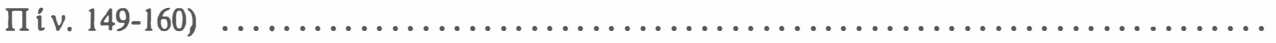

\section{IB' ЕФОРЕIA ПРО $\cdot\ulcorner\cdot \Sigma T O P I K \Omega N$ KAI K $\Lambda$ A $\Sigma I K \Omega N$ APXAIOTHT $\Omega N$}

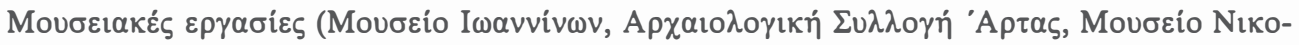




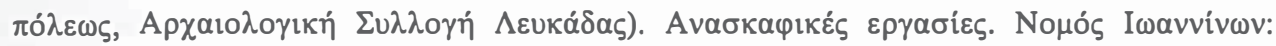

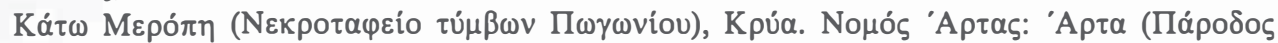

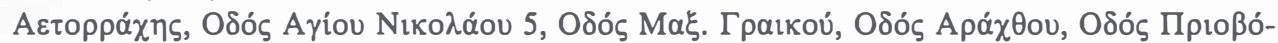

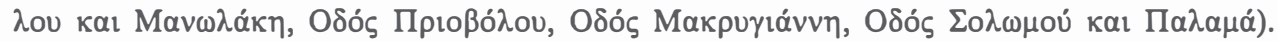

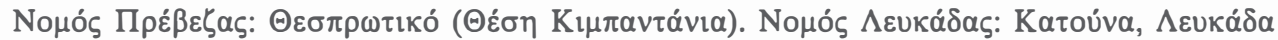

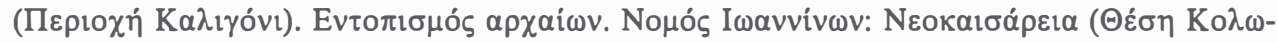

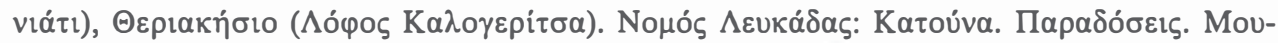

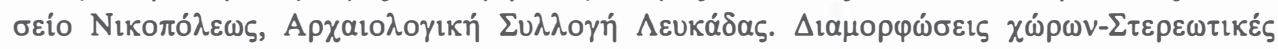

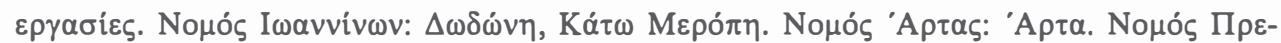

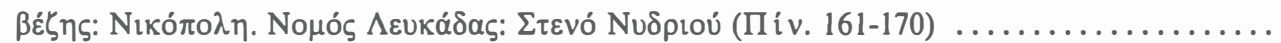

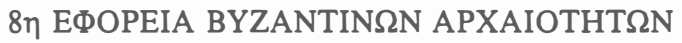

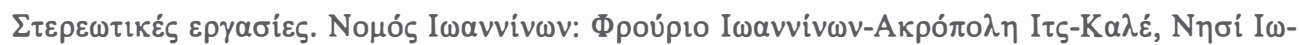

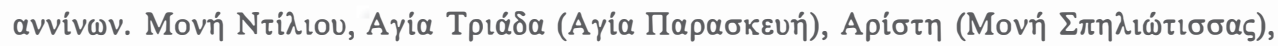

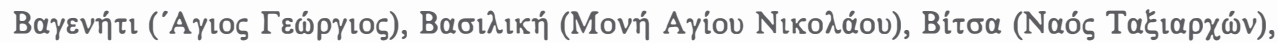
$\Delta \varepsilon \rho \beta i \zeta \zeta_{\imath} \alpha \alpha \alpha$ ('A

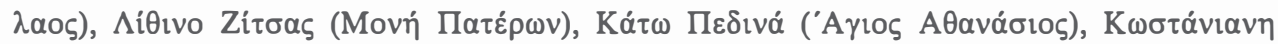

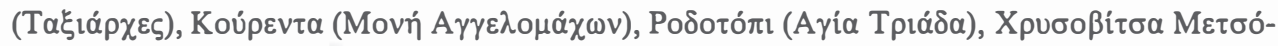

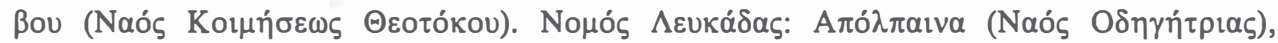

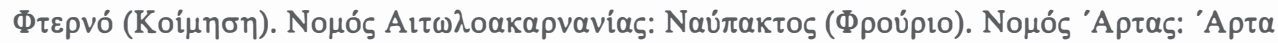

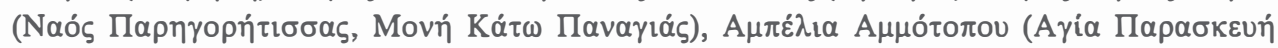

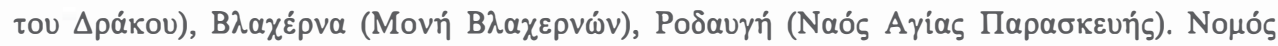

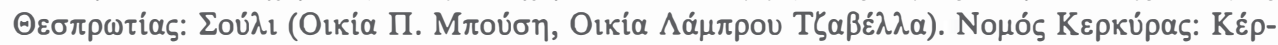

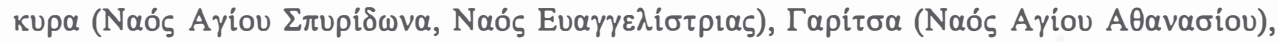

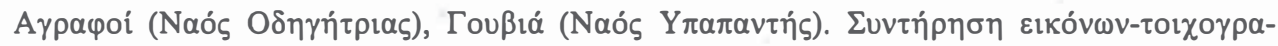

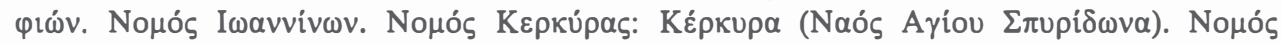

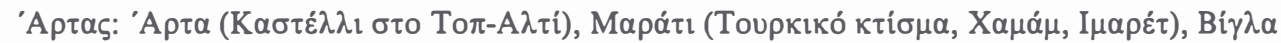

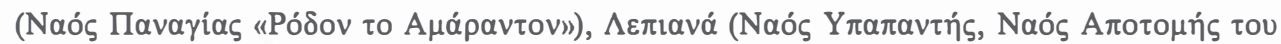

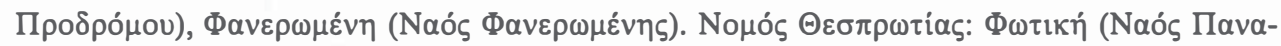

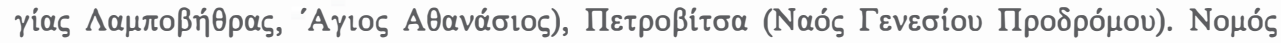

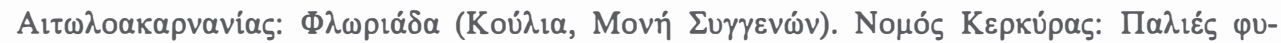

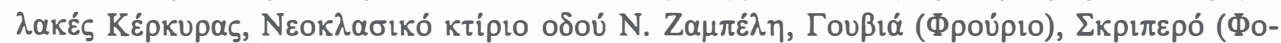

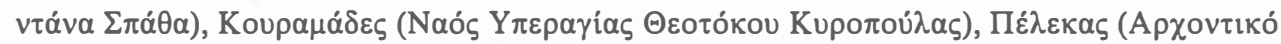

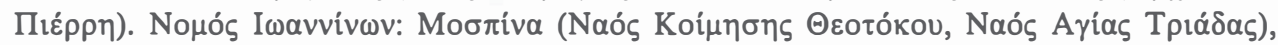

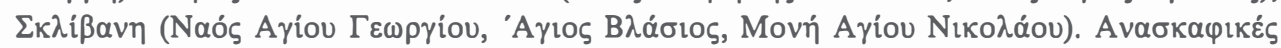

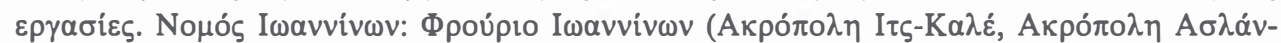

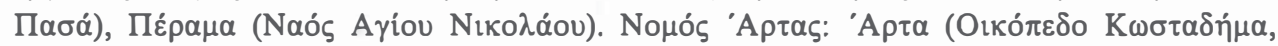

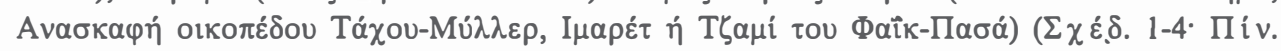
171-192)

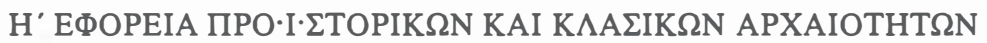

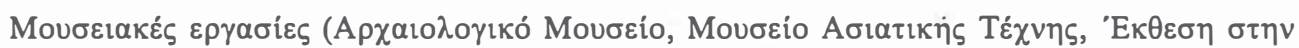

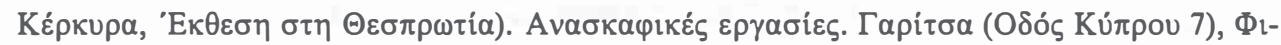

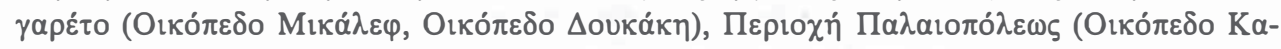

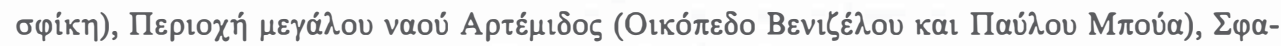

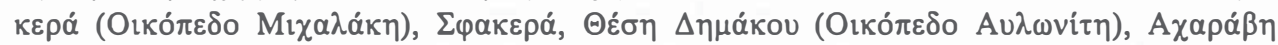

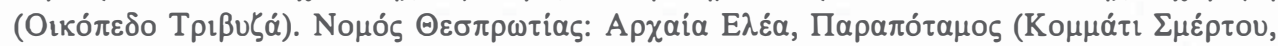




\section{$E \lambda \alpha \dot{\alpha} \varepsilon \alpha$}

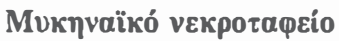

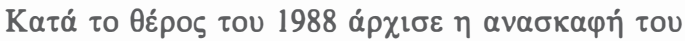

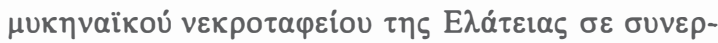
$\gamma \alpha \sigma i \alpha \tau \eta \varsigma \mathrm{I} \Delta^{\prime}$ Е

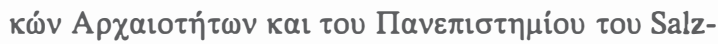

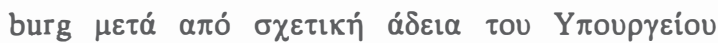

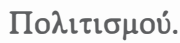

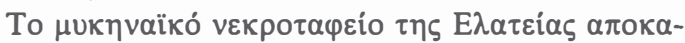

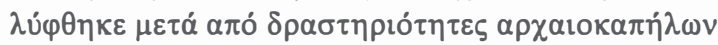

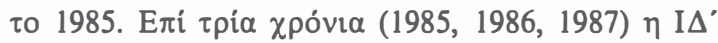

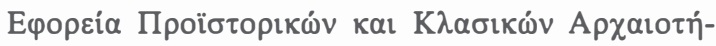

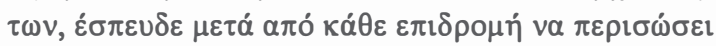

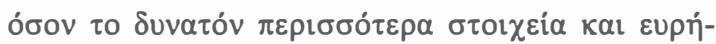

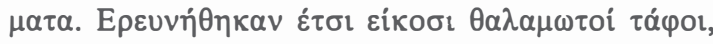

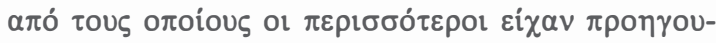
$\mu \varepsilon \dot{\varepsilon \omega \varsigma} \sigma u \lambda \eta \theta \varepsilon i^{40}$.

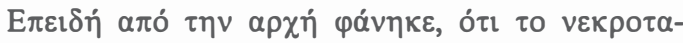

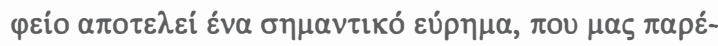

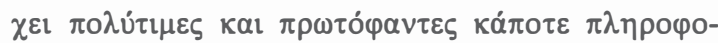

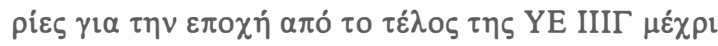

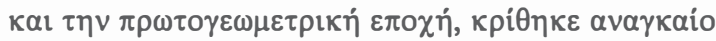

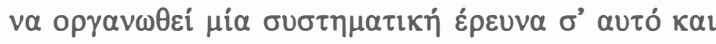

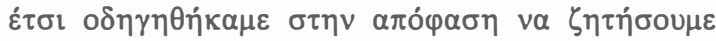

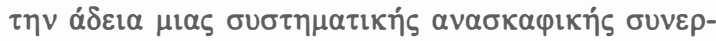

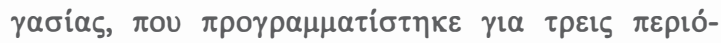

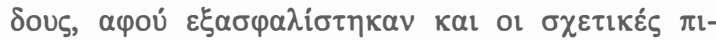

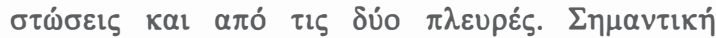

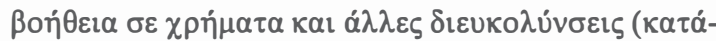

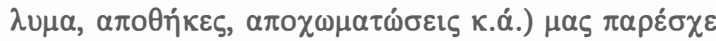

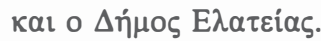

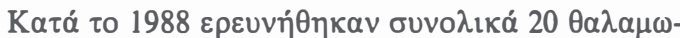

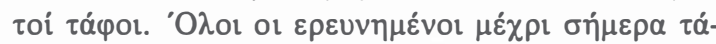

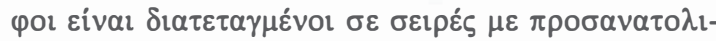

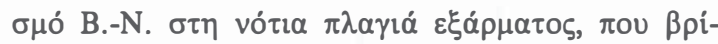

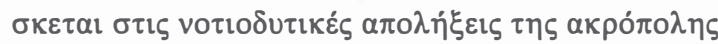

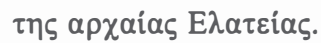

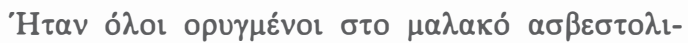

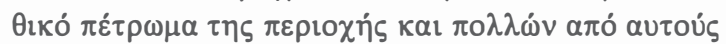

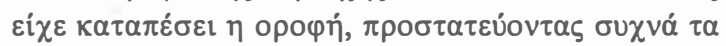

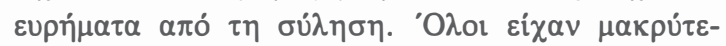

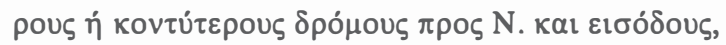

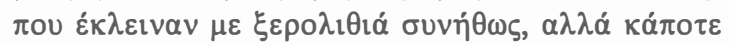
$\kappa \alpha \iota \mu \varepsilon \pi \lambda \alpha \dot{\alpha} \kappa \varepsilon \zeta \lambda i \theta \imath v \varepsilon \varsigma$.

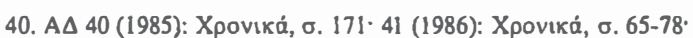
42 (1987): Xpovıкá, б. 231-234.

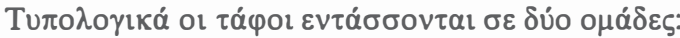
$O \mu \alpha ́ \delta \alpha \alpha$

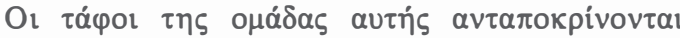

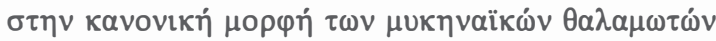
$\tau \alpha \dot{\alpha} \varphi \omega \nu, \mu \varepsilon \delta \rho o ́ \mu o-\sigma \tau o ́ \mu 10-\theta \alpha \dot{\lambda} \alpha \mu$ o ( $\Sigma \chi \varepsilon \dot{\varepsilon} \delta$. 14).

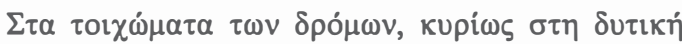

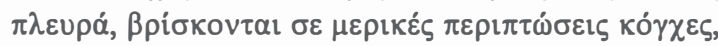

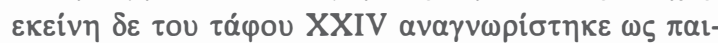

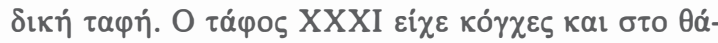

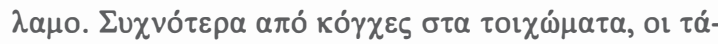

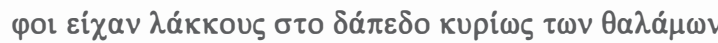

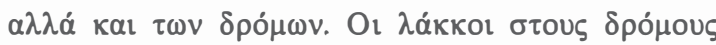

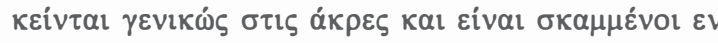

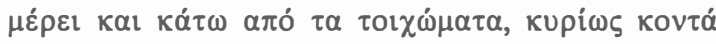

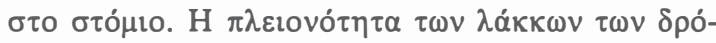

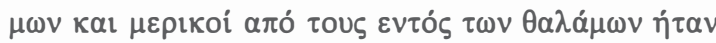

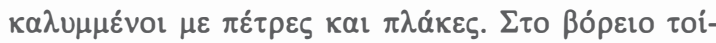

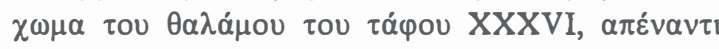

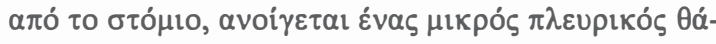
$\lambda \alpha \mu \circ \zeta$.

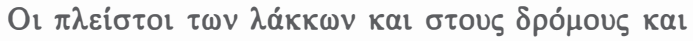

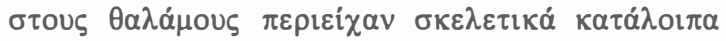

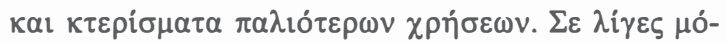

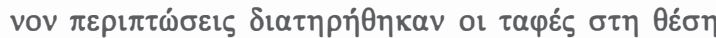

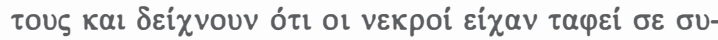

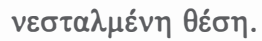

\section{$O \mu \alpha \dot{\delta} \alpha \beta$}

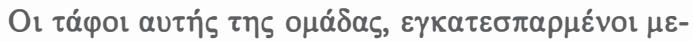

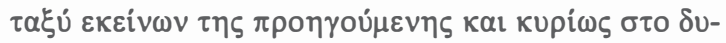

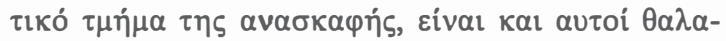

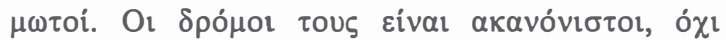
$\varepsilon \pi \iota \mu \varepsilon \lambda \omega ́ \varsigma ~ \lambda \alpha \xi \xi \varepsilon \cup \mu \varepsilon \dot{v o l ~ \kappa \alpha \iota ~ \sigma \tau o u \varsigma ~ \tau \alpha ́ \varphi o u s ~ X X X I ~ \kappa \alpha l ~}$

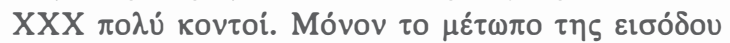

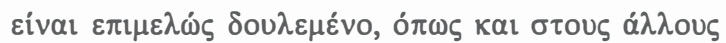

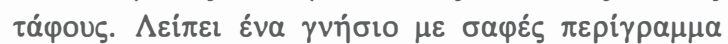

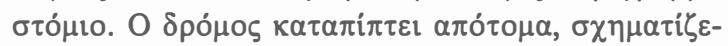

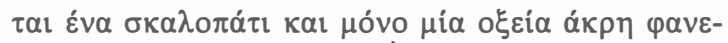

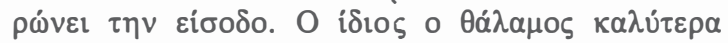

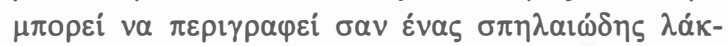

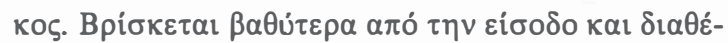

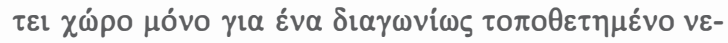

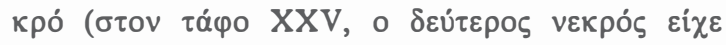

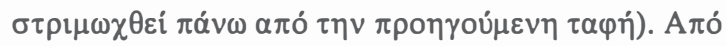

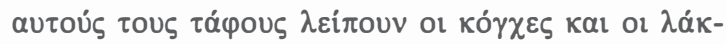

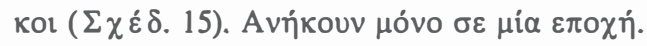

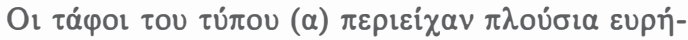

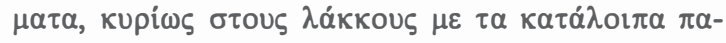

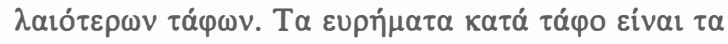
$\varepsilon \xi ̧ n \dot{~}$

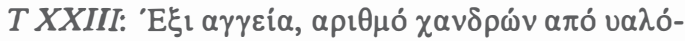

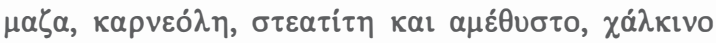

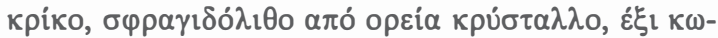



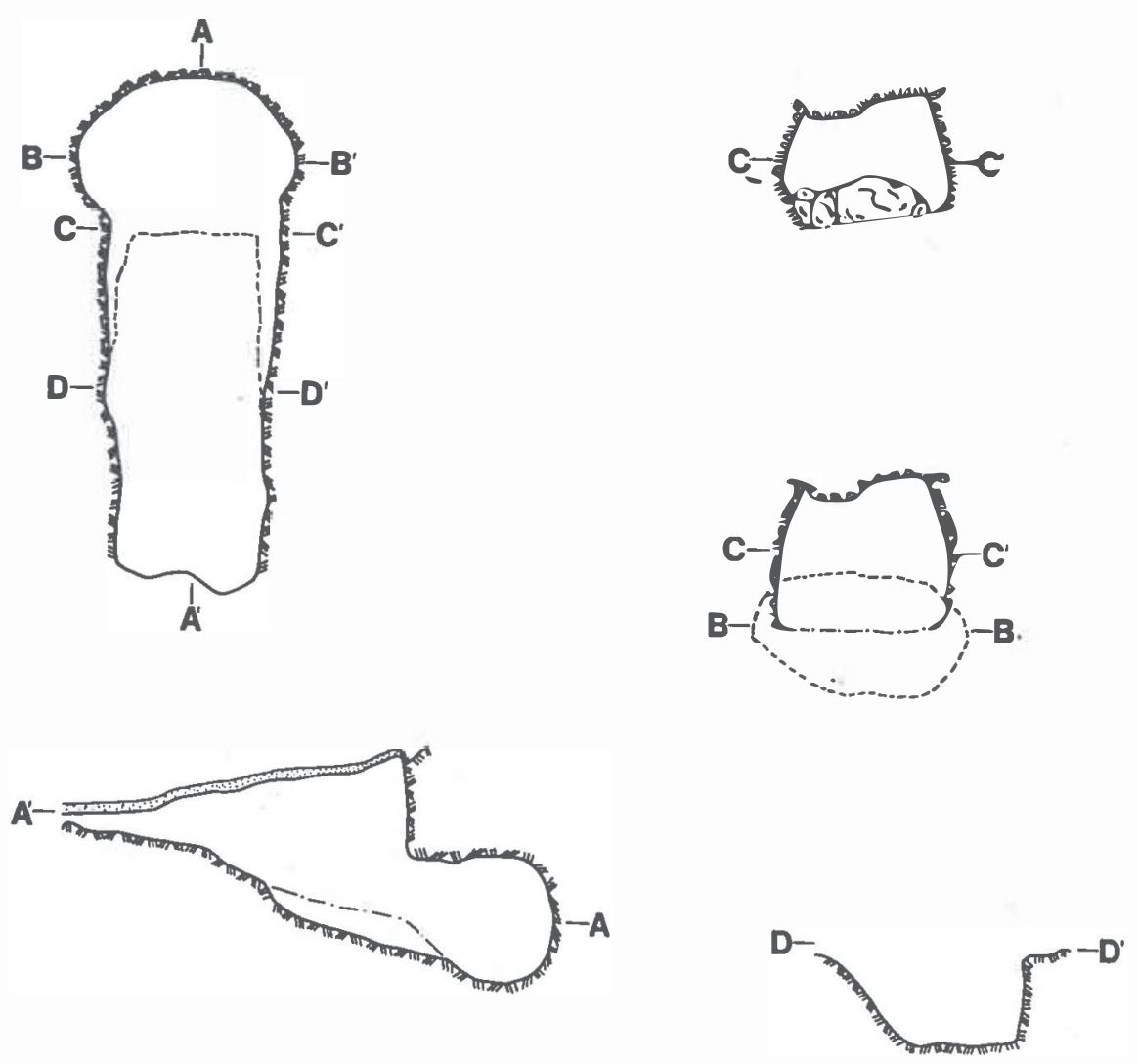

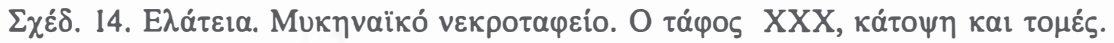

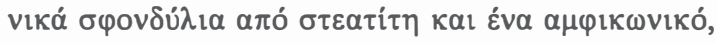

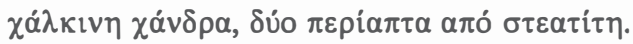

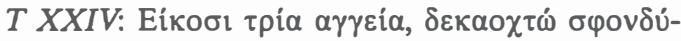

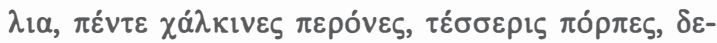

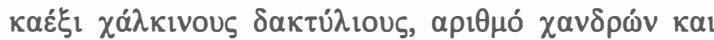

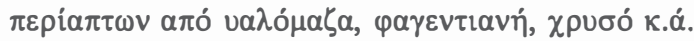

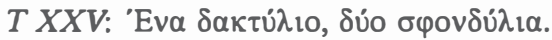

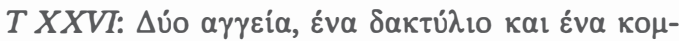

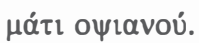

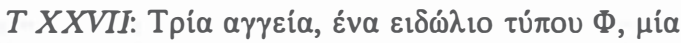

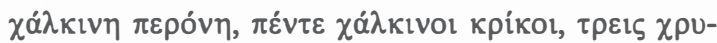

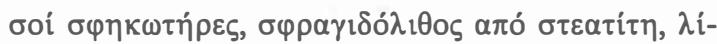

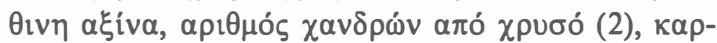

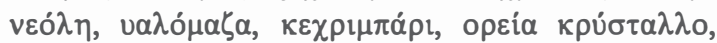

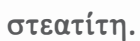

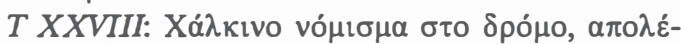

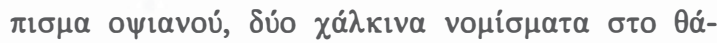

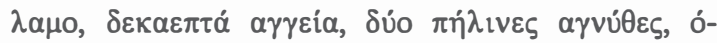

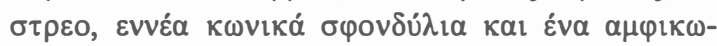

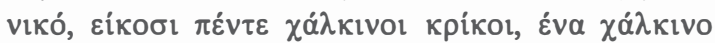

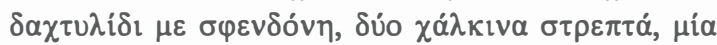

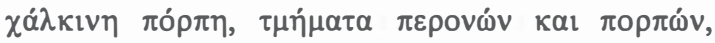

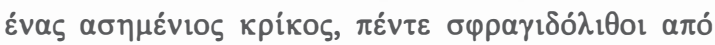

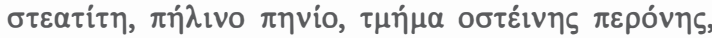

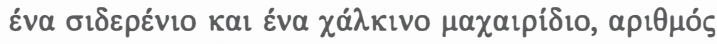

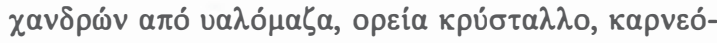

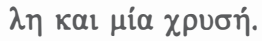

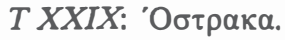

$T X X X:$ : 'Обтрака.

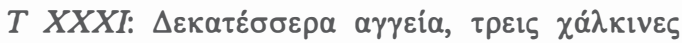

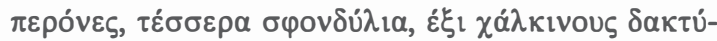

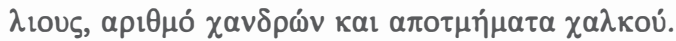

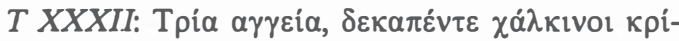

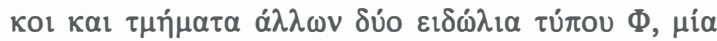

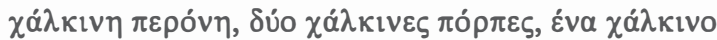

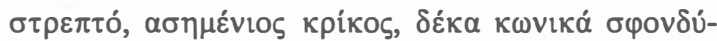

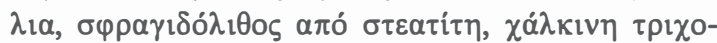

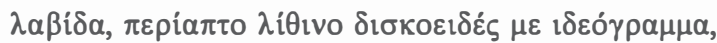

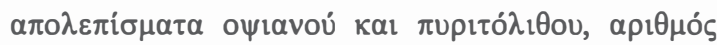

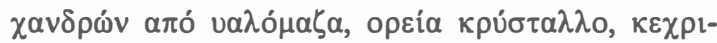
$\mu \pi \alpha ́ \rho l, \sigma \tau \varepsilon \alpha \tau i \tau \eta ~ \kappa \alpha l ~ \kappa \alpha \rho v \varepsilon o ́ \lambda \eta$.

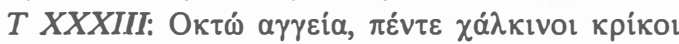

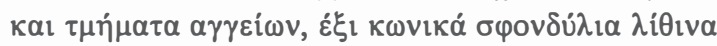

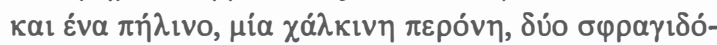

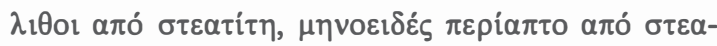

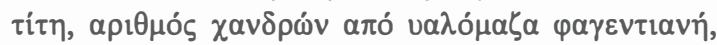



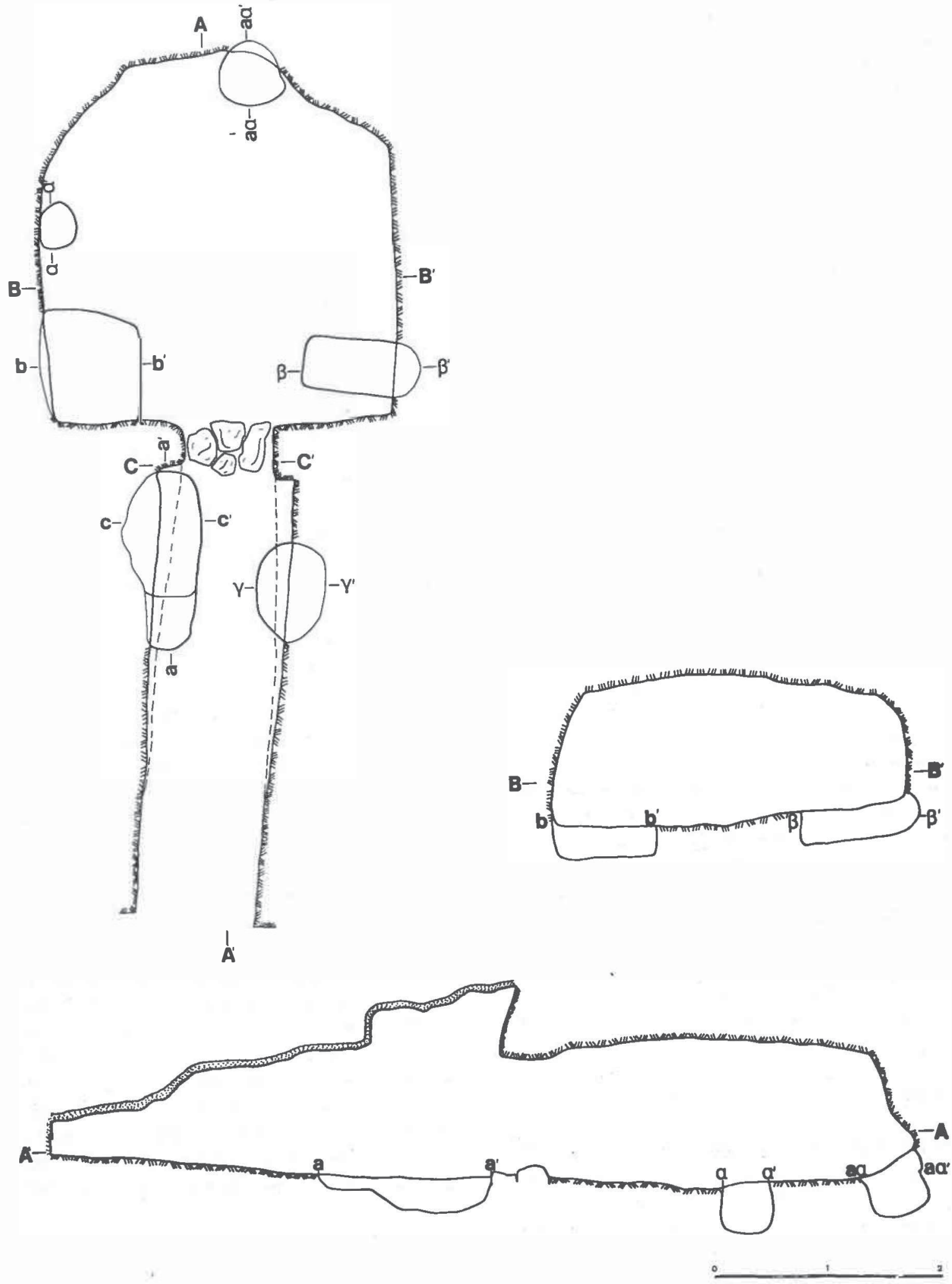

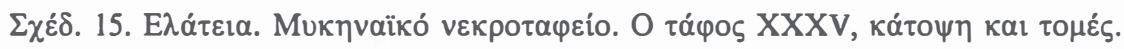




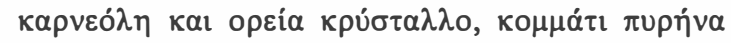
oభıavoú.

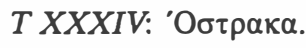

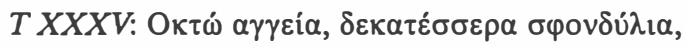

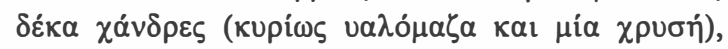

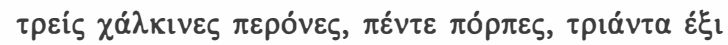

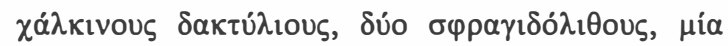

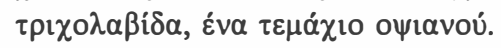

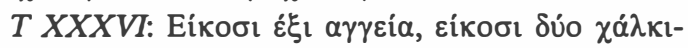

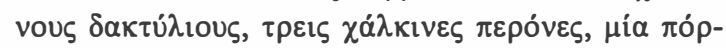

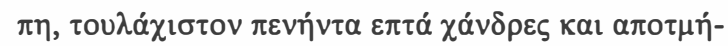

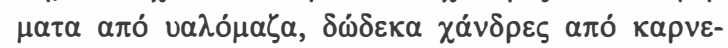

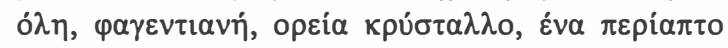

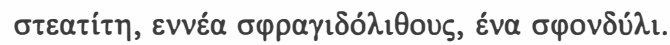

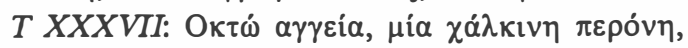

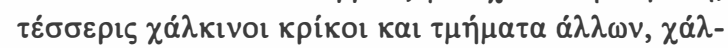

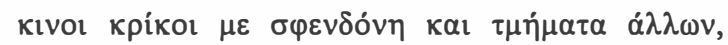

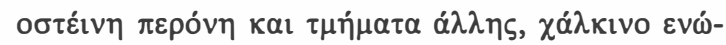

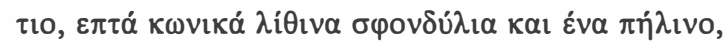

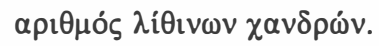

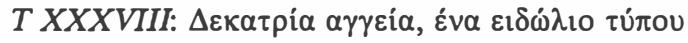

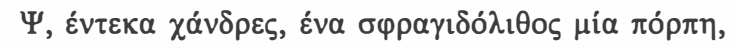

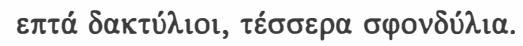

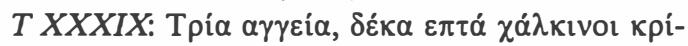

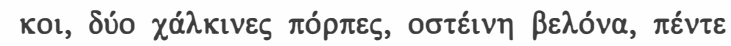

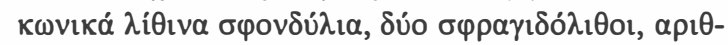

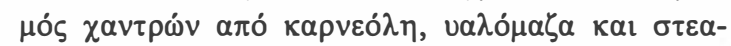

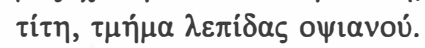

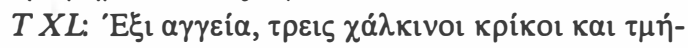

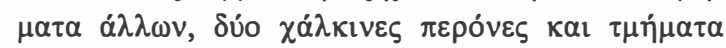

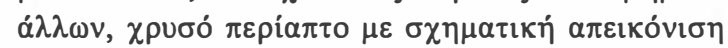

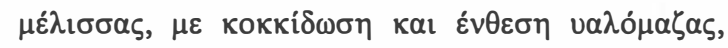

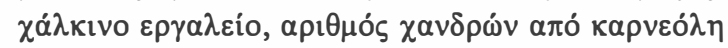

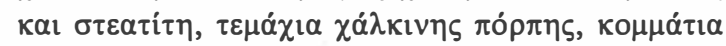

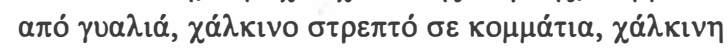

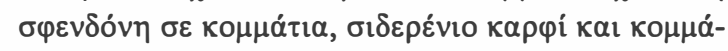
$\tau \iota \alpha \dot{\alpha} \lambda \lambda \omega v, \pi \circ \lambda \lambda \varepsilon \dot{\varepsilon} \varsigma \kappa \varepsilon \rho \alpha \mu i \delta \varepsilon \varsigma$.

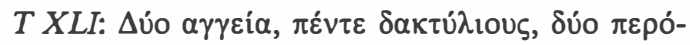

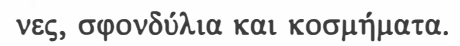

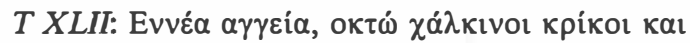

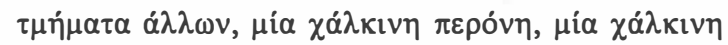

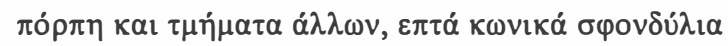
$\lambda i \theta v v \alpha, \mu i \alpha \alpha \alpha_{\alpha} v \tau \rho \alpha \alpha \pi$ ó $v \alpha \lambda o ́ \mu \alpha \zeta \alpha$.

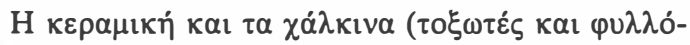

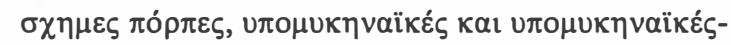

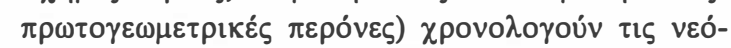

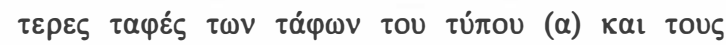

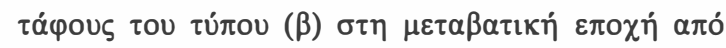

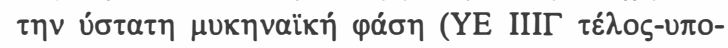

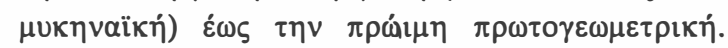

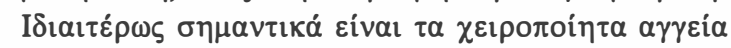

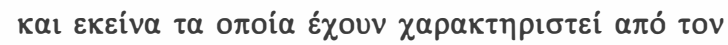

Schachermayer $\omega \varsigma$ « $\mu \varepsilon \tau \alpha \beta \alpha \tau \iota \kappa \alpha ́ »$ (Zwischenware).

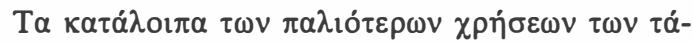

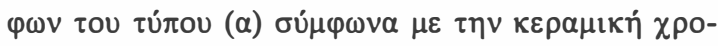

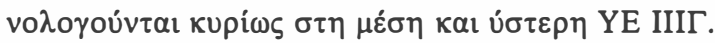

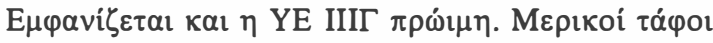
$\kappa \alpha \tau \alpha \sigma \kappa \varepsilon \cup \alpha \dot{\sigma} \sigma \eta \kappa \alpha \nu \sigma u ́ \mu \varphi \omega v \alpha \mu \varepsilon \tau \eta \nu \kappa \varepsilon \rho \alpha \mu \iota \kappa \eta ́ ~ \sigma \tau \eta \nu$ YE IIIB.

A

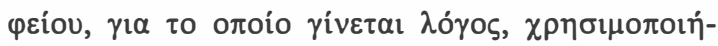

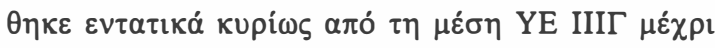

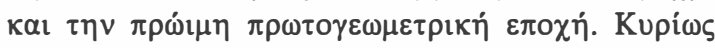

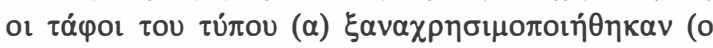

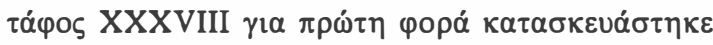

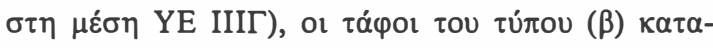

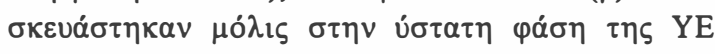
IIII.

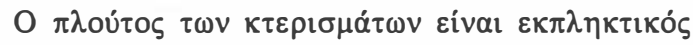

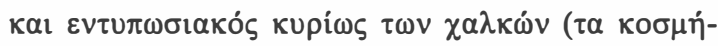

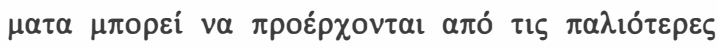

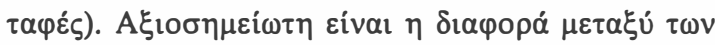

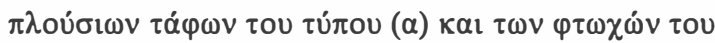

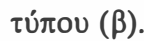

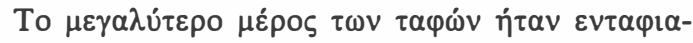

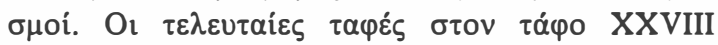

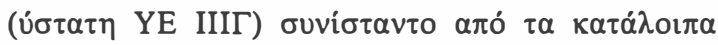

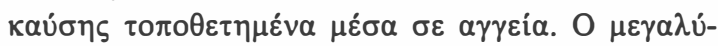

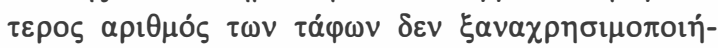

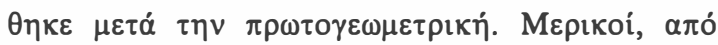

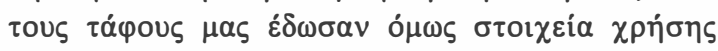

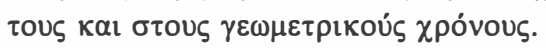

\section{Ф. $\triangle A K O P \Omega N I A$ - SIGRID DEGER-JALKOTZY}

\section{$\Pi \alpha v \alpha \gamma i \tau \sigma \alpha \mathrm{E} \lambda \alpha \dot{\alpha} \varepsilon \iota \alpha \varsigma$}

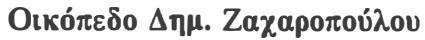

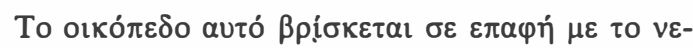

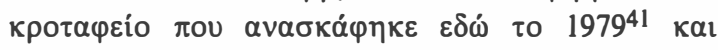

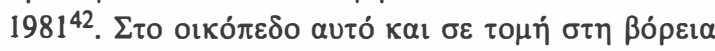

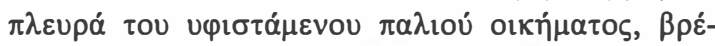

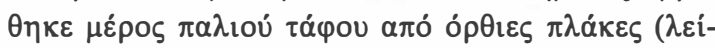

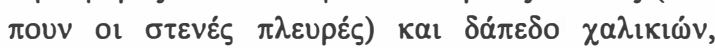

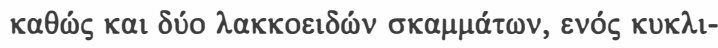

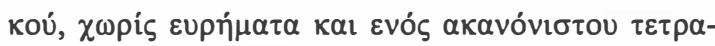

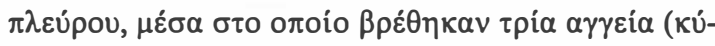

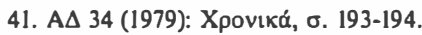

42. A $\Delta 36$ (1981): Xpovıкá, б. 221-222. 